\title{
Hand involvement in Charcot-Marie-Tooth disease type 1A may resemble rheumatoid arthritis
}

\author{
Meltem Alkan Melikoğlu \\ Department of Physical Medicine and Rehabilitation, Medical Faculty of Atatürk University, Erzurum, Turkey
}

Received: May 2016 Accepted: September 2016

A 18-year-old male patient was referred to our rheumatology clinic with pain and deformity in his hands. He reported minimal dysfunction, weakness, and sensory symptoms in addition to progressive pain and deformity in his hands for two years. On his physical examination, systemic evaluation was unremarkable and other joints were normal, as well as his feet. He presented hand deformities resembling rheumatoid arthritis (RA). Reversible ulnar deviation, metacarpophalangeal (MCP) joint flexion, proximal interphalangeal (PIP) joint extension, and distal interphalangeal (DIP) joint flexion (swan neck deformity) were observed in both hands. Adduction and opposition deformities were present in both thumbs, and all deformities in the right hand were more pronounced (Figures 1,2). There was no swelling, redness, or tenderness in his hands.

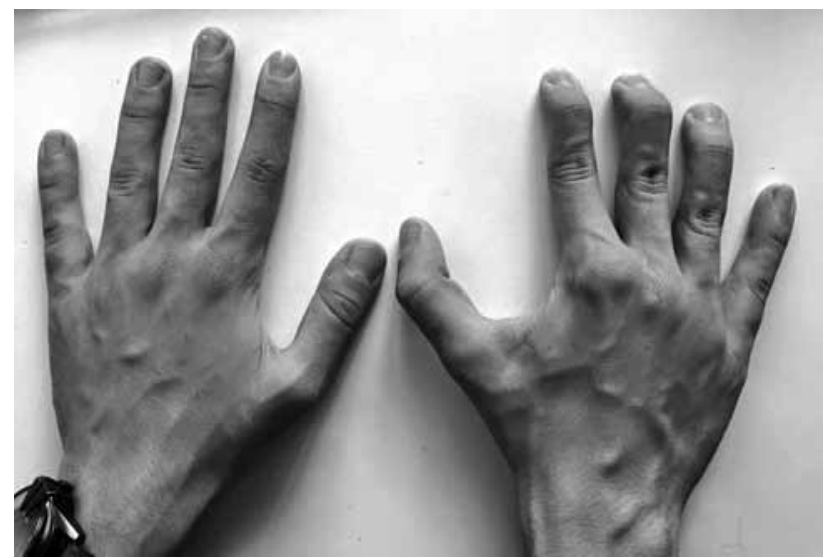

Figure 1. Hand deformity of the case with Charcot-MarieTooth disease type $1 \mathrm{~A}$ resembling rheumatoid arthritis.
Complete blood analysis and routine biochemical analyses were unremarkable. Rheumatoid factor, anti-citrullinated peptide (anti-CCP) and antinuclear antibodies (ANA) were negative. Radiographic findings of the hands were also normal. Based on his neurological symptoms, genetic tests were performed. He was diagnosed with Charcot-Marie-Tooth disease type 1A (CMT1A), depending on the duplication of the gene for peripheral nerve myelin protein 22 (PMP22) and clinical signs.

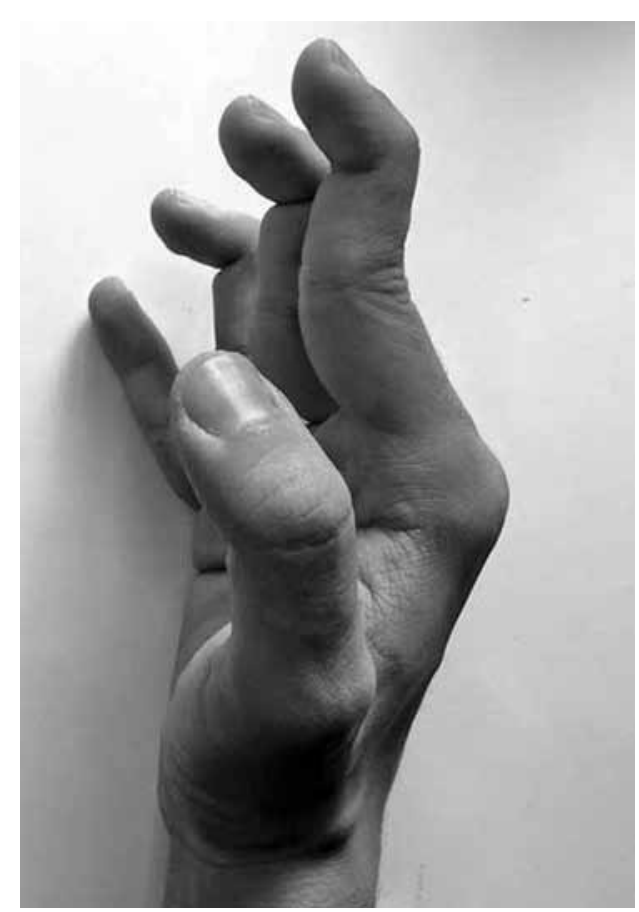

Figure 2. Hand involvement appearing a swan neck deformity. 
Charcot-Marie-Tooth disease is relatively common, inherited neuropathy with a prevalence ranging from 9/100,000 to $82 / 100,000$ worldwide. ${ }^{[1]}$ Charcot-MarieTooth disease type $1 \mathrm{~A}$, the most common form of $\mathrm{CMT}$, is a demyelinating neuropathy characterized by progressive length-dependent muscle weakness and atrophy. It predominantly affects lower extremities; therefore, foot and leg problems have been highly reported in the literature. However, its phenotype can be quite variable, which complicates the diagnosis and management. It has been also reported that hand problems are not uncommon in these patients; indeed, they may be present from the earliest stages of the disease, but may be underrecognized in early stages which leads to potential delay in treatment. ${ }^{[2]}$ The disease may also cause hand problems, such as dysfunction, sensory symptoms, pain, and deformity worsening with age. The deformity in hands may be variable and may be similar to RA, as in our case.

In conclusion, patients with CMT1, particularly with atypical phenotypes, may be sometimes difficult to recognize for physicians who are not familiar with the disease. We present this case to draw attention to this disease, which can be delayed for the correct diagnosis. An increased awareness of the disease may, therefore, contribute to earlier diagnosis.

\section{Declaration of conflicting interests}

The author declared no conflicts of interest with respect to the authorship and/or publication of this article.

\section{Funding}

The author received no financial support for the research and/or authorship of this article.

\section{REFERENCES}

1. Barreto LC, Oliveira FS, Nunes PS, de França Costa IM, Garcez CA, Goes GM, et al. Epidemiologic Study of Charcot-Marie-Tooth Disease: A Systematic Review. Neuroepidemiology 2016;46:157-65.

2. Burns J, Bray P, Cross LA, North KN, Ryan MM, Ouvrier RA. Hand involvement in children with Charcot-Marie-Tooth disease type 1A. Neuromuscul Disord 2008;18:970-3. 